\title{
Spatial distribution of oil in groundnut and sunflower seeds by nuclear magnetic resonance imaging
}

\author{
M R LAKSHMINARAYANA*, SYAMASUNDAR JOSHI**, \\ $\mathrm{G}$ A NAGANA GOWDA and $\mathrm{C}$ L KHETRAPAL; \\ Sophisticated Instruments Facility, Indian Institute of Science, Bangalore 560 012, India \\ *Department of Physics and **Department of Botany, University of Agricultural Sciences, \\ GKVK, Bangalore 560 065, India
}

MS received 15 October 1991; revised 16 January 1992

\begin{abstract}
The nuclear magnetic resonance imaging technique has been used to obtain images of different transverse and vertical sections in groundnut and sunflower seeds. Separate images have been obtained for oil and water components in the seeds. The spatial distribution of oil and water inside the seed has been obtained from the detailed analysis of the images. In the immature groundnut seeds obtained commercially, complementary oil and water distributions have been observed. Attempts have been made to explain these results.
\end{abstract}

Keywords. NMR imaging; oil distribution.

\section{Introduction}

Nuclear magnetic resonance (NMR) imaging is a recently innovated technique which can be employed to study, non-destructively, the spatial localizations of mobile proton pockets (Lauterbur 1973, 1974; Kumar et al 1975). This technique is being extensively employed in the study of intact biological systems relevant to medical diagnosis (Mansfield and Morris 1982). However, the applications to plant systems are still scarce; there are some reports of the applications to fruits (Wang et al 1988; Ishida et al 1989; Wang and Wang 1989), cereals (Song and Litchfield 1990), leaf (Walter et al 1989), roots (Matyac et al 1989), wood (Wang and Chang 1986; Chang et al 1989) and germinating seed (Connelly et al 1987). Differences in water binding have been measured in leaves under different conditions (Walter et al 1989). Detailed structure of an apple fruit has been obtained with emphasis on the water distribution (Wang et al 1988). The development of a core break down in 'Barlet pears has been investigated as a function of time and storage conditions (Wang and Wang 1989). In tomato fruit, water with long relaxation time is found to be accumulated preferentially in seeds and seed envelops in immature green fruit (Ishida et al 1989). In the mature tomato fruit, the total amount of water increases and water with long relaxation time is localized in the outer walls. There seems to be very little work on NMR imaging involving oil seeds.

In this communication, we report the $\left[{ }^{1} \mathrm{H}\right] \mathrm{NMR}$ imaging of oil seeds and the detection of spatial distribution of oil/water in them.

\section{Materials and methods}

Two different varieties of groundnut seeds viz., TMV- 2 (oil content $\sim 45 \%$ by wt)

$\overline{{ }^{\dagger} \text { Corresponding author. }}$ 
and Girnar-1 (oil content $\sim 38 \%$ by wt) and a sunflower variety Morden (oil content $\sim 32 \%$ by wt) were chosen for the investigations. The seeds contained low moisture content (about $8 \%$ ). In addition to the above, a commercially available variety of groundnut seed, obtained from the local market, with a high moisture content was also subjected to experimentation for purposes of comparison. In the commercial variety, therefore, it was possible to study the distribution of water as well as oil separately. On the other hand, in the TMV-2, the Girnar-1 varieties of groundnut seeds and the Morden variety of sunflower seed, only oil distribution was studied since the moisture content was low, NMR images were obtained with the help of a Bruker AMX-400 FTNMR spectrometer equipped with imaging facility and operating at $9 \cdot 4$ tesla. The bore size of the superconducting magnet was $89 \mathrm{~mm}$. Individual seeds were placed vertically with the radical end pointing up in a $10 \mathrm{~mm}$ glass tube surrounded by a radio frequency coil of $15 \mathrm{~mm}$ diameter. The proton images were obtained using chemical shift selective spin echo sequence (figure 1) exciting only oil resonances around $1.3 \mathrm{ppm}$ downfield with respect to tetramethylsilane protons. The slice thickness, echo time, repetition time and the number of data acquisitions were 500 micrometer, $8.7 \mathrm{~ms}, 1000 \mathrm{~ms}$ and 4, respectively for all the cases studied. Seven transverse slices with appropriate separations and one vertical slice at the centre were taken for all samples except for the commercial variety of the groundnut seed. The spatial resolutions in the image plane were $93.8 \times 93.8 \mu \mathrm{m}$ and $117.2 \times 117.2 \mu \mathrm{m}$ respectively for transverse slice and the vertical slice in the case of groundnut TMV-2; 78.1 $\times 78 \cdot 1 \mu \mathrm{m}$ and $117.2 \times 117.2 \mu \mathrm{m}$ respectively for transverse slice and vertical slice in the case of groundnut Girnar-1 and $46.9 \times 46.9 \mu \mathrm{m}$ and $78.1 \times 78.1 \mu \mathrm{m}$ respectively for transverse slice and vertical slice in the case of sunflower variety Morden. For the commercial variety of groundnut the spatial resolution was $93.8 \times 93.8 \mu \mathrm{m}$ for the transverse slice in the case of both oil and water images.

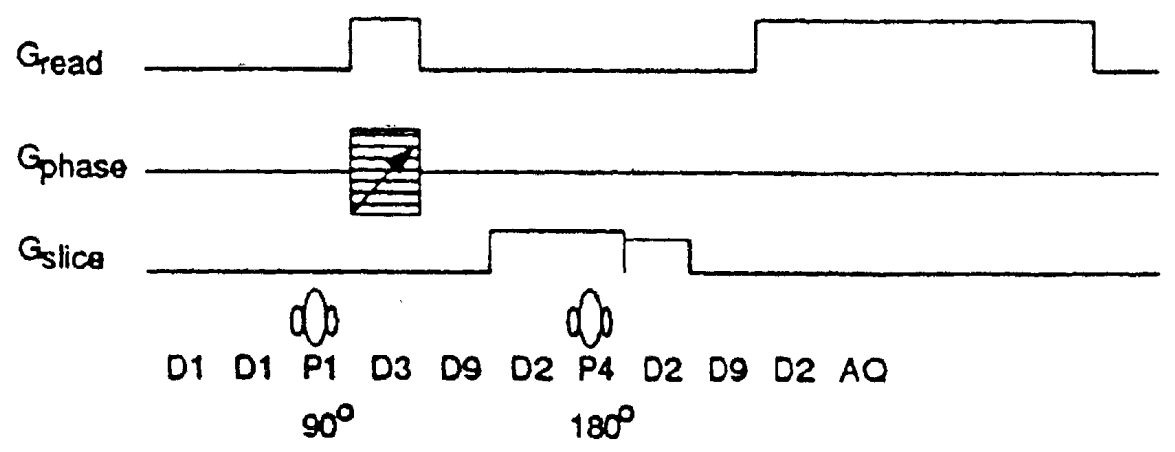

Figure 1. Chemical shift selective spin echo single slice pulse sequence. $G_{\text {read }}, G_{\text {phase }}$ and $G_{\text {slice }}$ are the corresponding gradients. $P_{1}$ and $P_{4}$ are the chemical shift. selective $90^{\circ}$ pulse and slice selective $180^{\circ}$ pulse respectively. $\mathrm{D}_{1}, \mathrm{D}_{3}, \mathrm{D}_{9}, \mathrm{D}_{2}$ and $\mathrm{AQ}$ describe repetition, phase encoding, echo time and gradient stabilization delays and data acquisition time respectively.

\section{Results and discussions}

The $\left[{ }^{1} \mathrm{H}\right]$ NMR spectra of intact oil seeds are shown in figure 2. The assignments of various peaks to the different functional groups in the triglyceride and also the 


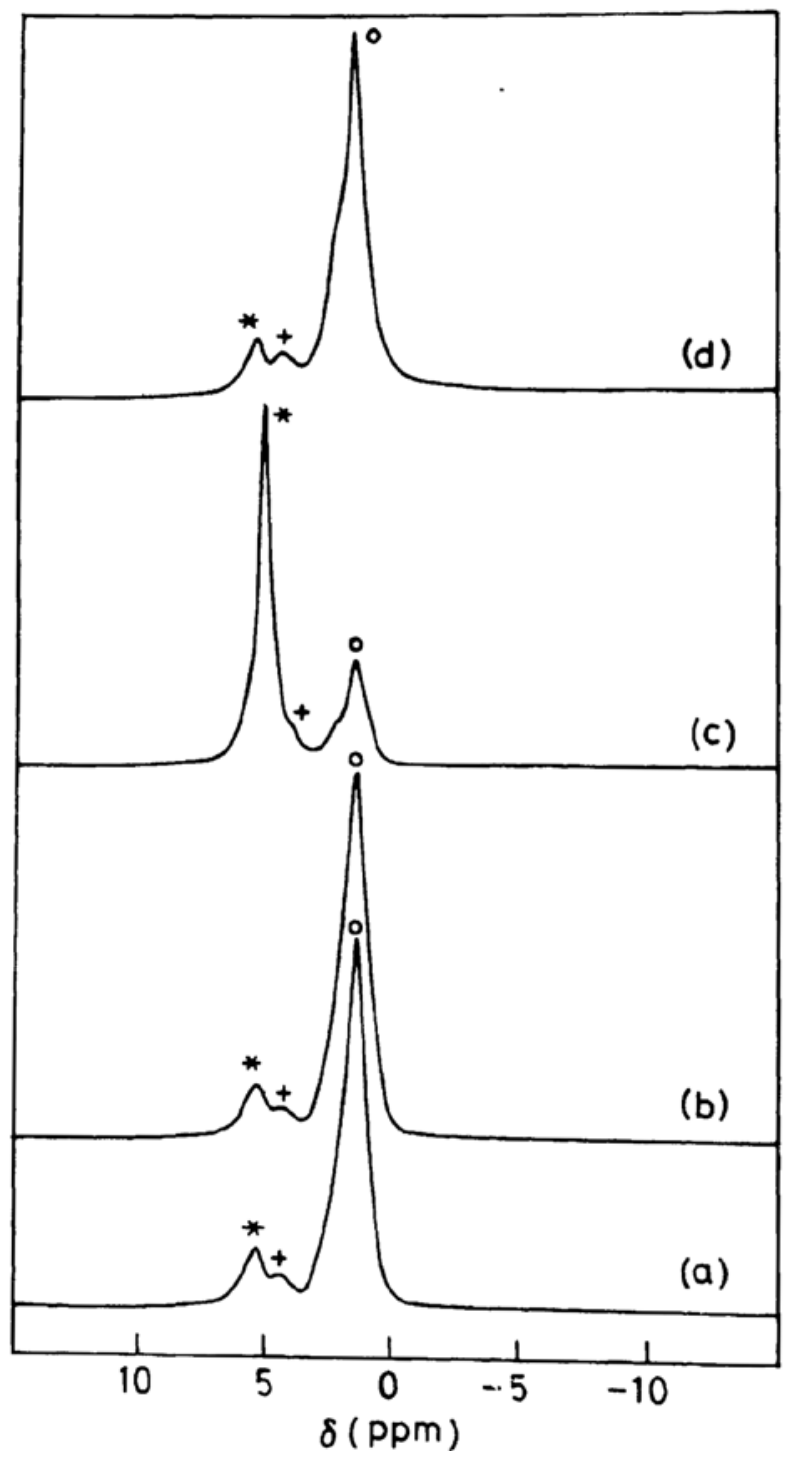

Figure 2. $\left[{ }^{1} \mathrm{H}\right] \mathrm{NMR}$ spectra of intact seeds, (a) Groundnut TMV-2. (b) Groundnut Girnar-1. (c) Commercially procured variety of groundnut, (d) Sunflower seed Morden. *, Water, olefinic protons and $\beta$-glyceride group.

,+ 4 glyceride protons.

o, Fatty acid chain $\mathrm{CH}_{2}$ protons.

position of water peak are well known (Convay and Johnson 1969). The intense signal at about $1.3 \mathrm{ppm}$ arising from a large number of $\left(\mathrm{CH}_{2}\right)$ groups in the triglyceride is representative of oil in the seed. If there is sufficient moisture in the seed a broad peak appears at about $5.0 \mathrm{ppm}$ with appropriate intensity. In the case of commercially procured groundnut seed there was a strong water peak (figure $2 \mathrm{c}$ ) compared to that for the TMV-2 and Girnar-1 varieties (figure $2 \mathrm{a}, \mathrm{b}$ ). In addition 
the peaks due to water, olefinic protons and $\beta$-glyceride proton could not be resolved (figure 2). For the quantitative interpretation of oil/water distribution in the seeds the scale is provided on the right hand side of the image as shown in figures 3-5. The red region corresponds to the maximum oil/water followed by yellow and blue. The pink region at the bottom of the scale corresponds to the lowest oil content. Thus in the oil picture of the seeds, the distribution of oil in the seed is indicated by the colour distribution in the NMR image, in the case of groundnut seeds, the seed material and inner air space between the two cotyledons are very clearly seen in their respective images of both the transverse and the vertical sections (figure 3 ). The intensity distribution shows a very clear pattern wherein the adaxial region of the cotyledon is red and yellow and the abaxial region is blue. This means that the oil is stored in the seed to a greater extent in the adaxial region than in the abaxial region (Bewley and Black 1978, 1982). In fact, a sharp gradient in the oil concentration between the two regions is indicated within the seed from the adaxial region to the abaxial region of the entire seed. This inference is arrived at from the fact that the oil images of the various transverse sections and the vertical section show characteristic colour distribution. The two

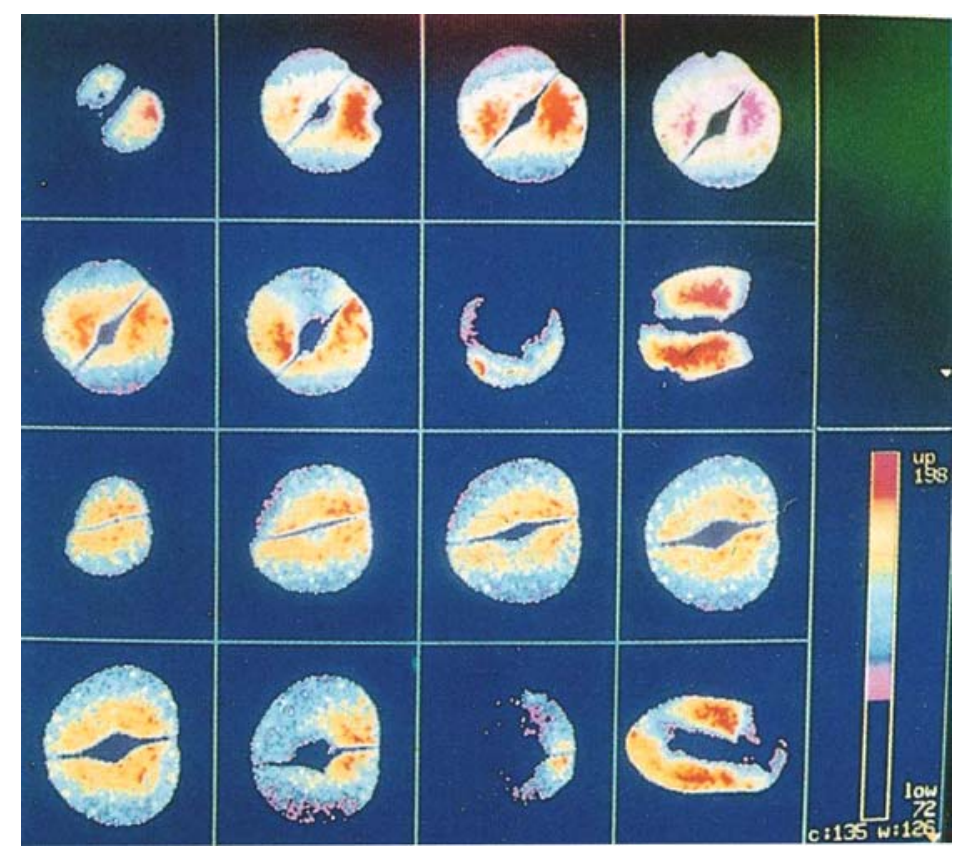

Figure 3. Horizontal and vertical sections of the $\left[{ }_{1} \mathrm{H}\right]$ NMR oil images of two varieties of groundnut viz., TMV-2 (two top rows) and Girnar-1 (two bottom rows). First row: from left to right respectively the images are from the slice $5 \cdot 5,4 \cdot 0,2.5$ and $1.0 \mathrm{~mm}$ below the centre of the seed. Second row: from left to right, the respective images are from the slices at the centre and those 1.0 and $2.5 \mathrm{~mm}$ above the centre. The extreme right image on the second row is due to the central vertical section. Third row: from left to right, the respective images are $5 \cdot 5,4 \cdot 0,2.5$ and $1.0 \mathrm{~mm}$ below the centre. Fourth row: the extreme left image is due to central cross section and the next two images are from slices at 2.5 and $4.0 \mathrm{~mm}$ above the centre. The extreme right image is due to central vertical section. The field of view was $12 \mathrm{~mm}$ and the read, phase and slice gradients used were $9 \cdot 79,5.45$ and $14.09 \mathrm{G} / \mathrm{cm}$. 
sets of oil images corresponding to the groundnut varieties TMV-2 and Girnar-1 exhibit quite distinctly different colour patterns in the adaxial regions of the cotyledons. This can be interpreted on the basis that the two groundnut varieties do not possess the same oil content. Infact, TMV-2 contains about $45 \cdot 0 \mathrm{wt} \%$ oil while Girnar contains about $38.0 \mathrm{wt} \%$. An analysis of the colour distribution in the oil images of various horizontal and vertical sections in the sunflower seed variety Morden yields similar information though not exactly the same (figure 4). The oil is more localized at adaxial region than in the abaxial region. This is in agreement with the information obtained by optical microscopic techniques following the conventional procedural protocol like fixing, sectioning and staining of the seeds. The palisade parenchyma in the adaxial region of the cotyledons contain more lipid than the abaxial spongy cells (Bhasha 1982). Similar contrasting microenvironment is perhaps prevalent in the cotyledons of groundnut seed as well, since there is a resemblance in the NMR oil images of both sunflower and groundnut seeds. The imaging experiment performed on the commercially available variety of groundnut seed yielded two images corresponding to oil and water obtained in the same transverse section of the seed. The two images exhibit distinctly different features indicative of two entirely different intracellular environments in the two cotyledons (figure 5). It is seen that the oil is present predominantly in one of the cotyledons and water in the other. A visual examination of the seed indicated that the seed was immature. Thus it can be interpreted that in this specific immature groundnut seed

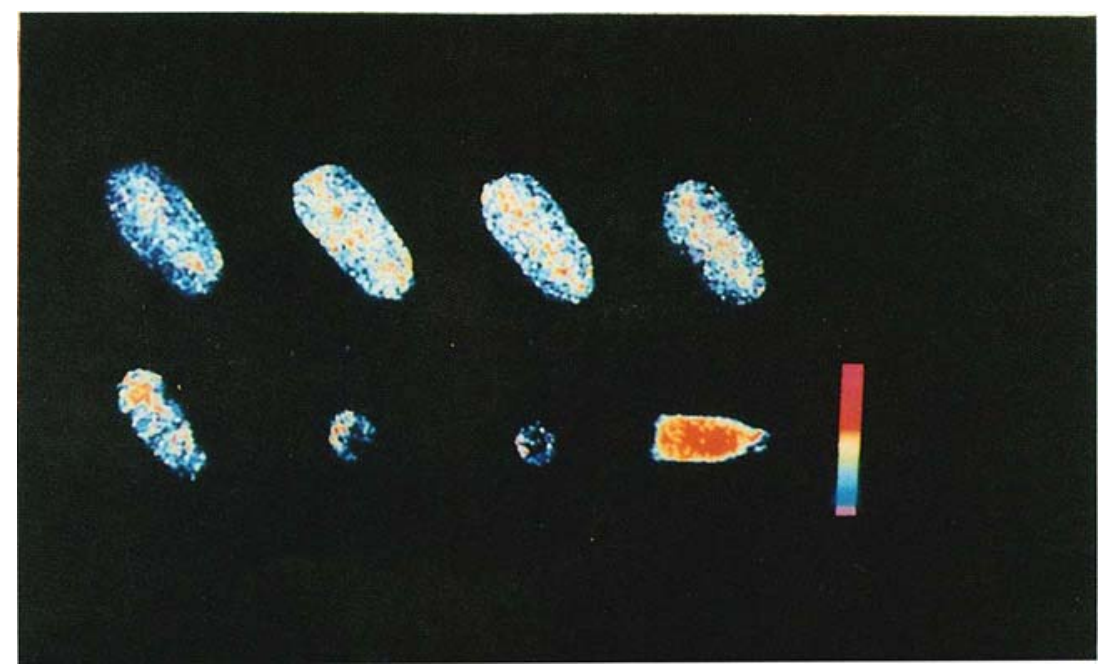

Figure 4. Horizontal and vertical sections of the $\left[{ }^{1} \mathrm{H}\right] \mathrm{NMR}$ oil images of a Morden variety of sunflower seed. First row: from left to right, the images are from 2.5 and $1.0 \mathrm{~mm}$ below the centre. The next image is of the central cross section and the extreme right image is due to the slice $1.0 \mathrm{~mm}$ above the centre. Second row: the respective images are $2.5,3.5$ and $4.0 \mathrm{~mm}$ above the centre. The extreme right image of this row is the central vertical cross section. The field of view was $6 \cdot 0$ and $10.0 \mathrm{~mm}$ for the transverse and vertical slices respectively. The read, phase and slice gradients were $11.75,6.54$ and $14.09 \mathrm{G} / \mathrm{cm}$ respectively for transverse sections and 19.58, 10.90 and 14.09 G/cm, respectively for the vertical slice. 


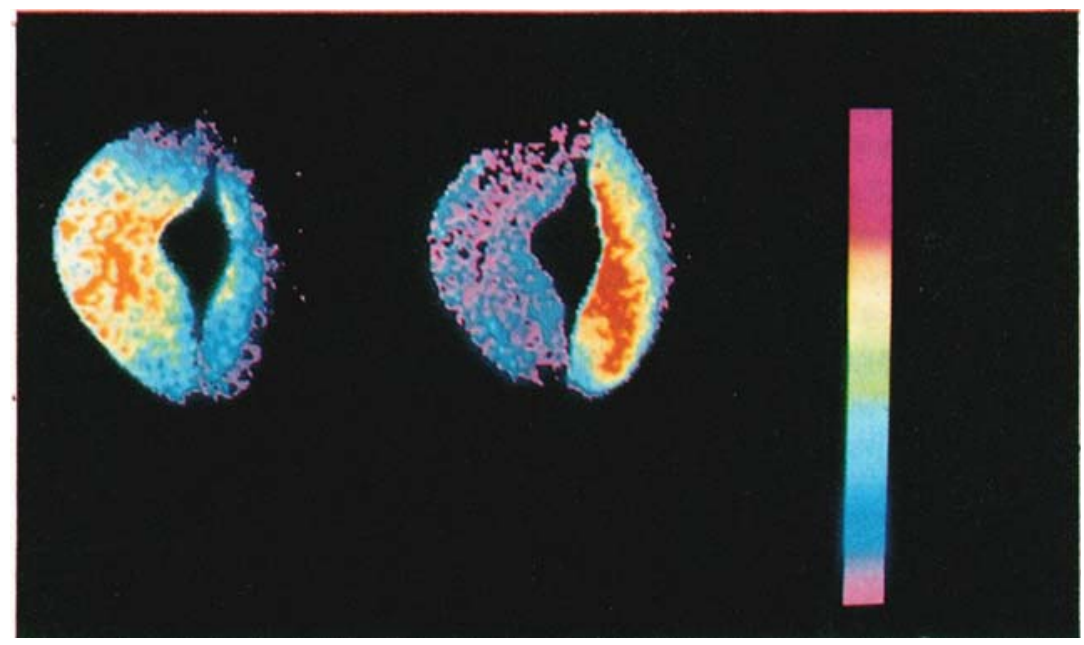

Figure 5. $\left[{ }^{1} \mathrm{H}\right]$ NMR images of transverse section of commercial variety of groundnut seed. Left: water image; right: oil image. The field of view was $12.0 \mathrm{~mm}$ and, the read, phase and slice gradients were $9 \cdot 79,5.45$ and $14.09 \mathrm{G} / \mathrm{cm}$ respectively.

where the oil build-up is incomplete, the oil and water distribution within the seed is not at all uniform but is preferentially localized in a complimentary manner. The water soluble sugar yet to be converted to storage lipids are confined to one of the cotyledons. A physiological disorder may be responsible for this differential micro environment present in the two cotyledons.

Further work on the NMR imaging of germinating oil seeds in order to investigate the physiological events that follow transport processes of the bulk fraction in living tissues during early stages of germination and the study of the oil build-up in developing seeds is in progress.

\section{Conclusions}

The results demonstrate the applications of the NMR imaging technique for the study of the oil/water distribution in the intact seeds. Such a technique offers great potential for the study of the oil build-up/breaking in developing/germinating seeds.

\section{References}

Bewley K E and Black M 1978, 1982 Physiology and biochemistry of seeds in relation to germination Vols I and II (Heidelberg: Springer-Verlag)

Bhasha K E 1982 Histological and histochemical studies in the viable and nonviable seeds of sunflower (Helianthus annuus-L) subjected to germination, M Sc thesis, University of Agricultural Sciences, Bangalore

Chang S J, Olson J R and Wang P C 1989 NMR imaging of internal features in wood; For. Prod. J. 39 43-49

Connelly J A, Lohman B, Loughman B C, Quiquompox H and Ratcliffe R G 1987- High resolution imaging of plant tissues by NMR; J. Exp. Bot. 38 1713-1723 
Convay T F and Johnson L F 1969 Nuclear magnetic resonance of oil unsaturation in single corn kernels; Science 164 827-828

Ishida N, Kobayashi T, Koizumi, M and Kauno H $1989{ }^{1} \mathrm{H}-\mathrm{NMR}$ imaging of tomato fruits; Agric. Biol. Chem. 53 2363-2367

Kumar A, Welti D and Ernst R R 1975 NMR Fourier zeugmatography; J. Magn. Reson. 18 69-83

Lauterbur P C 1973 Image formation by induced local interactions. Examples employing nuclear magnetic resonance; Nature (London) 242 190-191

Lauterbur P C 1974 Magnetic resonance zeugmatography; Pure Appl. Chem. 40 149-157

Mansfield P and Morris P C 1982 NMR imaging in biomedicine (New York: Academic Press)

Matyac C A, Cofer G P, Bailey J E and Johnson G A 1989 In situ observations of root-gall formation using nuclear magnetic resonance imaging; J, Nematol, 21 131-134

Song H and Litchfield J B 1990 NMR imaging of transient 3-dimensional moisture distribution in an ear of corn during drying; Cereal Chem. 67 580-584

Walter L, Balling A, Zimmermann U, Haase A and Kuhn W 1989 Nuclear magnetic resonance imaging of leaves of Mesembryanthemum crystallinum L, Plants grown at high salinity; Planta 178 524-530

Wang C Y and Wang P C 1989 Non-destructive detection of core breakdown in 'Bartlef pears with nuclear magnetic resonance imaging; Hortic. Sci. 24 106-109.

Wang P C and Chang S J 1986 Nuclear magnetic resonance imaging of wood; Wood Fiber Sci. 18 308314

Wang S Y, Wang P C and Faust M 1988 Non-destructive detection of water core in apple with nuclear magnetic resonance imaging; Sci. Hortic, 35 227-234 